\title{
STANDALONE APPLICATION USING JAVA AND ANFIS FOR PREDICTING ELECTRIC ENERGY CONSUMPTION BASED ON FORECASTED TEMPERATURE
}

\author{
Dragan Mlakić, Srete Nikolovski \& Dean Vucinic
}
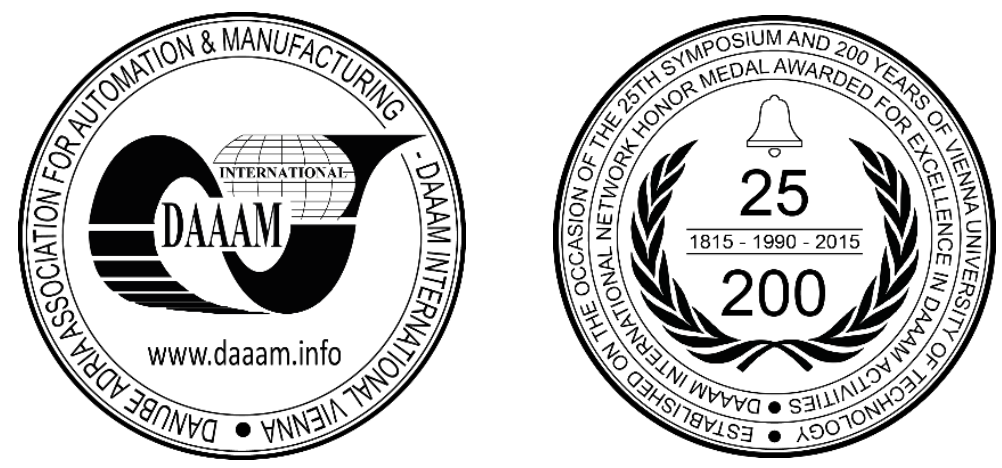

This Publication has to be referred as: Mlakic, D[ragan]; Nikolovski, S[rete] \& Vucinic, D[ean] (2016). Standalone Application Using JAVA and ANFIS for Predicting Electric Energy Consumption Based on Forecasted Temperature, Proceedings of the 27th DAAAM International Symposium, pp.0671-0677, B. Katalinic (Ed.), Published by DAAAM International, ISBN 978-3-902734-08-2, ISSN 1726-9679, Vienna, Austria

DOI: $10.2507 / 27$ th.daaam.proceedings.097

\begin{abstract}
Predicting energy consumption is the ability, which is useful anywhere where the energy resource is the main product. In particular, the electric energy is a very expensive product to be wasted without a concrete need. The need for fast prediction of the electric energy consumption is based on various inputs covering the following aspects: any time, any sampling resolution, any depth based on well-known and available data. Today, the advancing of the technology allows the new generation of software applications to integrate different methods for the prediction of the electric energy consumption, which is based on various available data coming from anywhere. Focus of this paper is flexibility of AI methods in predicting energy consumption and availability of input data for predicament. In this paper, the method for predicting el. energy consumption is based on a daily temperature using the Adaptive Neuro Fuzzy Inference System (ANFIS). Comparison of results are presented in paper between ANN and ANFIS on same input data, and that puts ANFIS method as best for predicting energy consumption. The results analysis is done with the developed MatLab software and the data acquisition module is specifically developed in JAVA. The data source is the web service available as "weather underground" web service. All the components are integrated in a performing and compact lightweight application that supports mobility, easy to use and easy to change functionality. The modelling, testing and validation are made on the data acquired from the electric energy consumption provided by the distribution system of the town on Kreševo in Bosnia and Hercegovina.
\end{abstract}

Keywords: ANFIS; Fuzzy; JAVA; electric energy consumption; MatLab; Simulink; application; mobility.

\section{Introduction}

The use of the Artificial Intelligence (AI) methods for predicting the electric energy consumption is becoming an ongoing trend, which has recently started. Many papers, researches and ideas have emerged and presented interesting work based on ANN, Fuzzy logic, GA, FA, and weather conditions gathered from many different places [1]. Today. The weather data are available from the web services, like wunderground.com, weather.com, intellicast.com, etc., which are 
accurate and diverse data to be reused. The capturing of those data and using them as input to the AI methods for predicting the energy consumption is not a new idea [2] [3] [4] [5] [6] [7]. Bringing them to a higher level of abstraction and make them available on any system and at any time, through a simple GUI, which nicely brings together highly modular and complex software tool is the focus of this research paper. Performing fast analysis, with mobility and easiness of use, are enabled through different set of tools. The importance of the energy consumption forecasting, in this case, is based on the weather forecast input, as a very important parameter for avoiding suboptimal management of an energy network, and for dispatcher to control in advance the load or unload of the network, and in such way to prevent the unnecessary outages or waste of the costly and valuable electrical energy.

In praxis, it is very important to make fast prediction of the consumption for the next custom period, as sampled in this use case for the distribution area of the town Kreševo located in Bosnia and Herzegovina. It is well known that every time is needed to perform finding, segmenting, preparing, loading, training, testing and presenting data. In order to reduce time and simplify such work it was essential to make further analysis, like management of line breakers in distribution network and power flow without risk of outage or energy waste [6].

Many research papers have been applying the AI methods for predicting the electric energy consumption based on variety of data input [2] [3] [4] [5]. Some papers compare the AI methods to find the most appropriate method to calculate such results. By considering the results of those papers, the Artificial Neural Network (ANN) is among the best and most accurate methods found for such forecasting and predicting [9] [10] [11].

In this paper, ANN is compared to the Adaptive Neuro Fuzzy Inference System (ANFIS), which is validated on the same input data according to the measured data from the Automatic Meter Reading (AMR) system, which is installed on the sampled electric energy network in the area of Kreševo. For the comparison of these results the best method was chosen between ANFIS and ANN, and used for the application implemented with the MatLab GUIDE. The JAVA programming language is used for gathering the data from the web service wunderground.com, based on the direct link giving access to the forecasting weather results.

The JAVA application reads the entire web page by recognising the temperature data needed for ANFIS in order to predict the consumption. The buffer for storing the input data is generated as *.txt file. The output data are also stored in the next generated *.txt file for further use, which file size is unlimited. The flexibility of and multiplatform ability of such JAVA application are the reasons for the development of the data gathering application in the selected JAVA language. The call-back for the JAVA application module is done within the GUIDE application, and based on the user choice.

The result of this applied approach is a fast, accurate and flexible application with an intuitive GUI and opening variety of choices for the end user

\section{Electric energy distribution for the area of Kreševo}

The geographic area, for which the forecasting is conducted is completely measurable on every giving/receiving point between the other areas, and data are already calculated in format of $\mathrm{kWh}$, with resolution which is available for the daily or monthly forecasting. The Automatic Meter Reading (AMR) is conducted with the system Automatic Meter Management (AMM) based on information taken from meters in every substation within the area of Kreševo, having the secondary voltage level of $35 / 10 \mathrm{kV}$ transformer in the energy receiving point located in the substation $\mathrm{TS} 35 / 10 \mathrm{kV}$ Kreševo. One point is border between area Kreševo and area Kiseljak. Fig. 1. shows a single line diagram for the distribution area of Kreševo with the energy receiving points marked at the border point with area Kiseljak. The energy consumption of area Kreševo is calculated using (1):

$$
E=E_{R}-E_{K i s}
$$

Where is:

$E_{R}$ - energy received to area Kreševo;

$E_{K i s}$ - energy supplied to area Kiseljak;

$\mathrm{E}$ - energy we seek to forecast.

Most of the time the area of Kreševo is supplying energy to the area of Kiseljak, thus it is not taken into the consideration the situation, when energy is coming from Kiseljak to Kreševo, as those situations are rare. The gathered measured data from AMR/AMM, are input data for creating forecasting methods, ANFIS and ANN, developed in Matlab. 


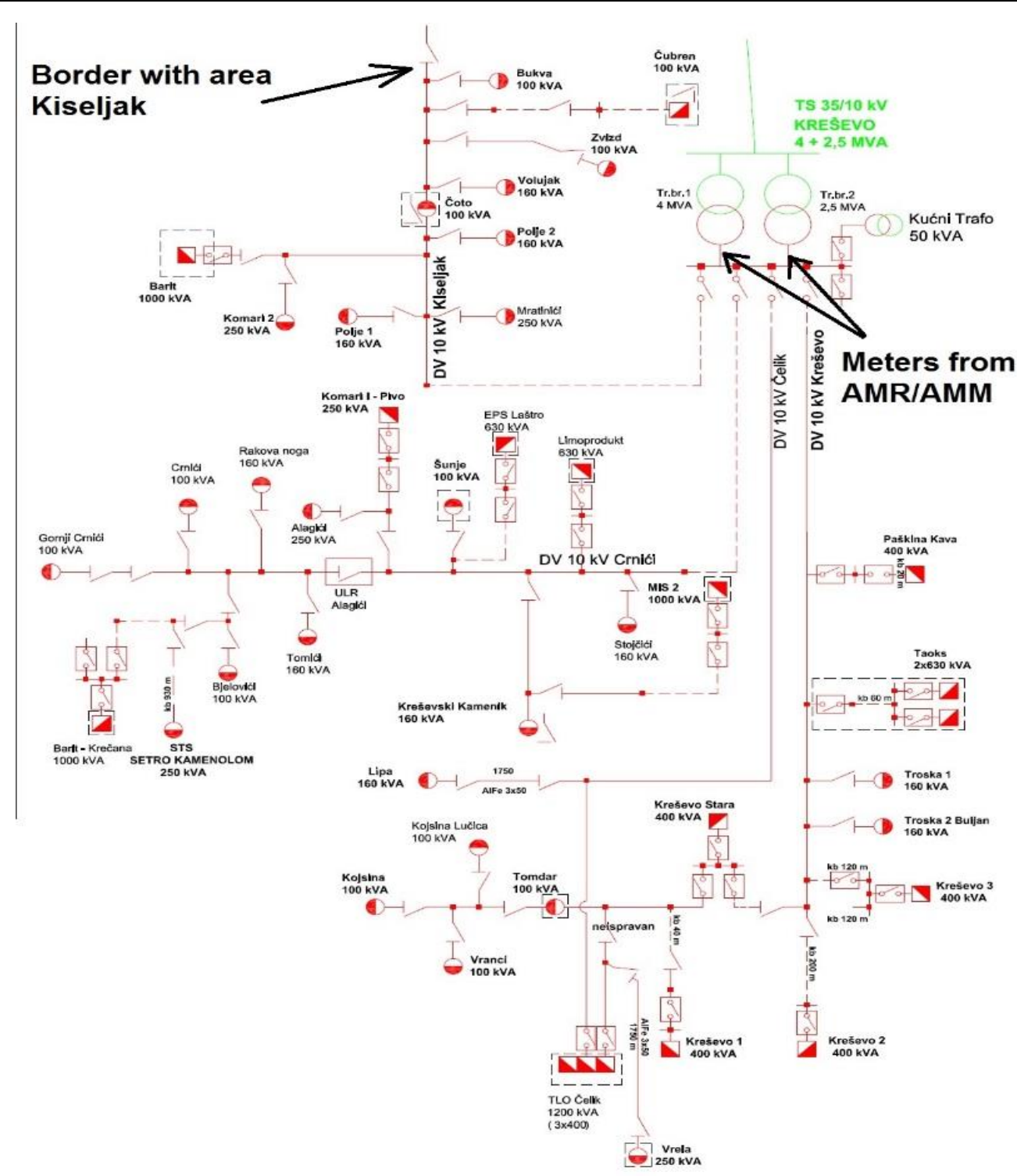

Fig. 1: Single line diagram of the distribution area of Kreševo.

\section{Forecasting methods}

Methods for forecasting the energy consumption were discussed through many conferences, research papers, and articles [7] [9] [10] [11]. Most of those papers discuss the accuracy and complexity of the proposed methods, which are based on their mathematical models, requirements for training, and checking results [8] [9] [12]. It was noticed that a few papers have in focus the elegancy and effectiveness of those methods applied to the everyday praxis. In this paper two methods were discussed and compared with same input data and tested according to measured values from AMR system. The input data are maximum, average and minimum temperature, acquired for one day within the targeted month. Those data are joined with the measured values of consumed electric energy during that day of the targeted month. By considering such data and combine it with, weather temperature and energy consumption, the method for forecasting the next month consumption is created and analysed.

\subsection{ANFIS}

The Adaptive Neuro Fuzzy Inference System is widely used in recent time and already introduced in these papers. In this case, the created ANFIS tool has 3 inputs and 1 output, with the Sugeno decision type, and the Gauss membership functions. Inputs for its training consists of 257 samples, and the average error of measured data is around $9 \%$. The created rules are 64, defuzzy method is weighted as average since based on the Sugeno decision type. The tool for training, testing and validating ANFIS is Matlab Fuzzy module. The generated rules are shown in Fig. 2, and surface of created ANFIS is shown in Fig.3. 


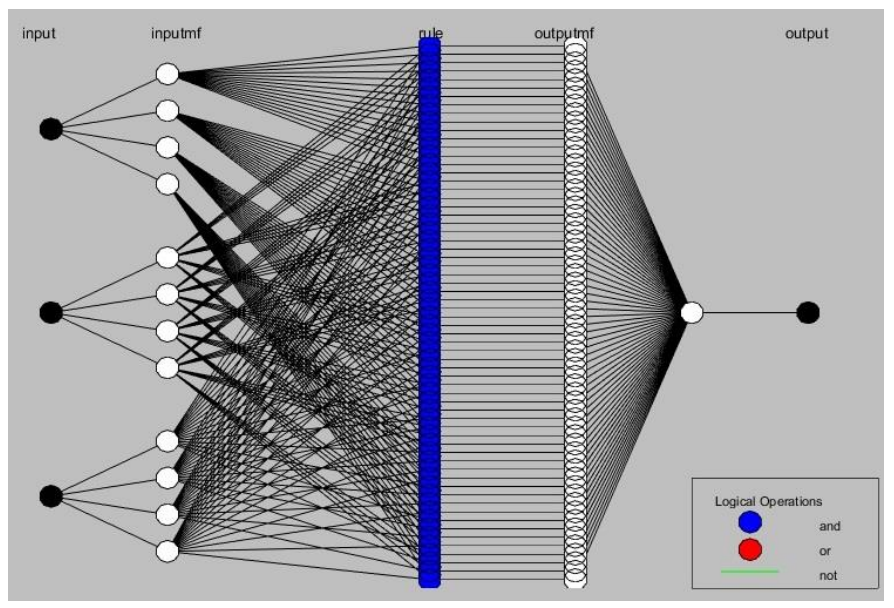

Fig. 2. Trained ANFIS for forecasting electric energy consumption based on weather online data.

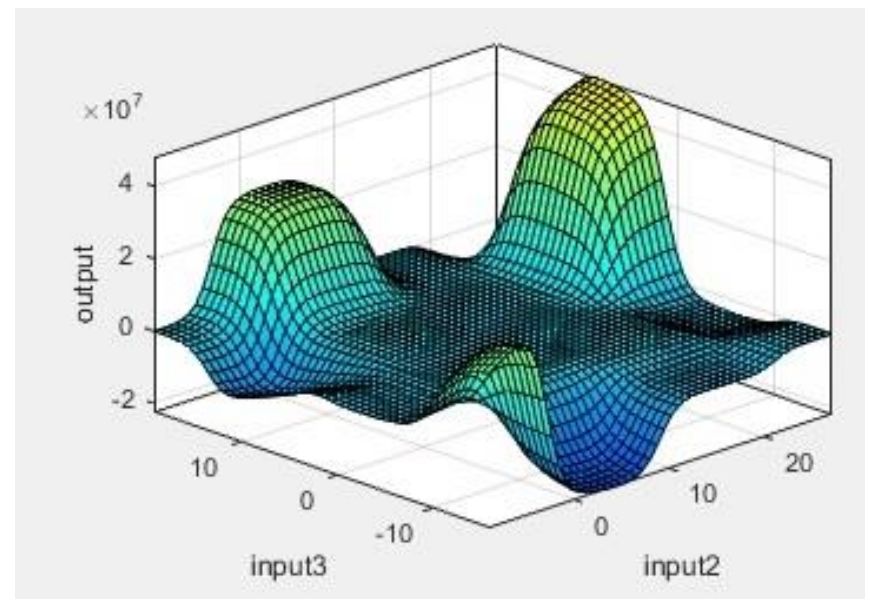

Fig. 3. Surface of created ANFIS.

\subsection{Artificial Neural Network}

The second method used is ANN in form of the Neural Network Fitting Tool from Matlab. The introduction of ANN is done in previous papers [11]. The Matlab nftool is used for creating neural fitting function with 365 samples, 3 inputs, 1 output, 10 hidden layers, training function is Bayesian Regularization method, performance measured by Mean Squared Error, and the calculations are done by using the MEX function. Fig. 4 shows Error Histogram for the creation process of ANN. Fig 5. Shows regression of created ANN function.

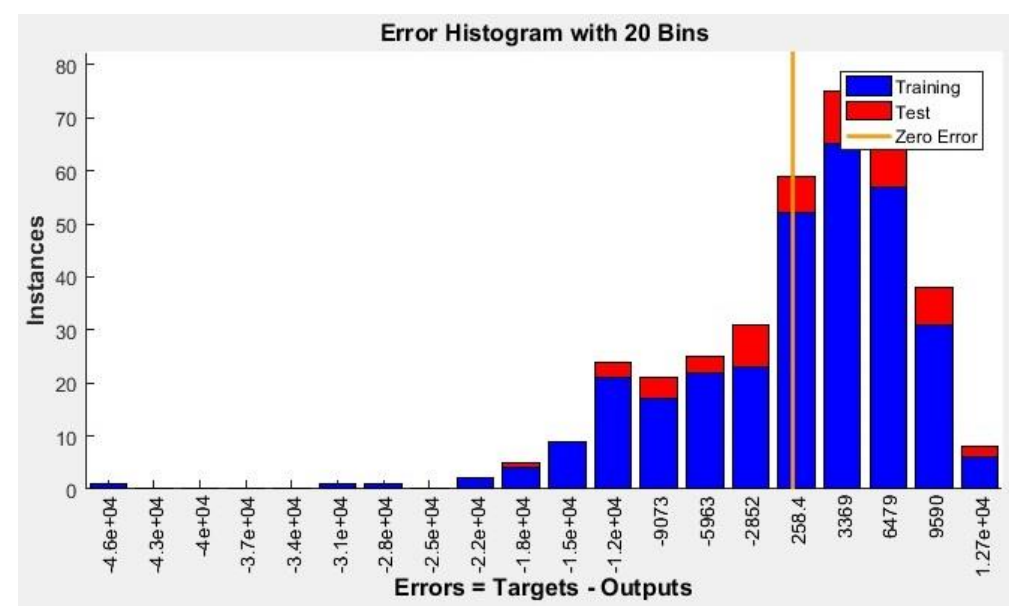

Fig. 4. Error histogram for created Neural Network. 

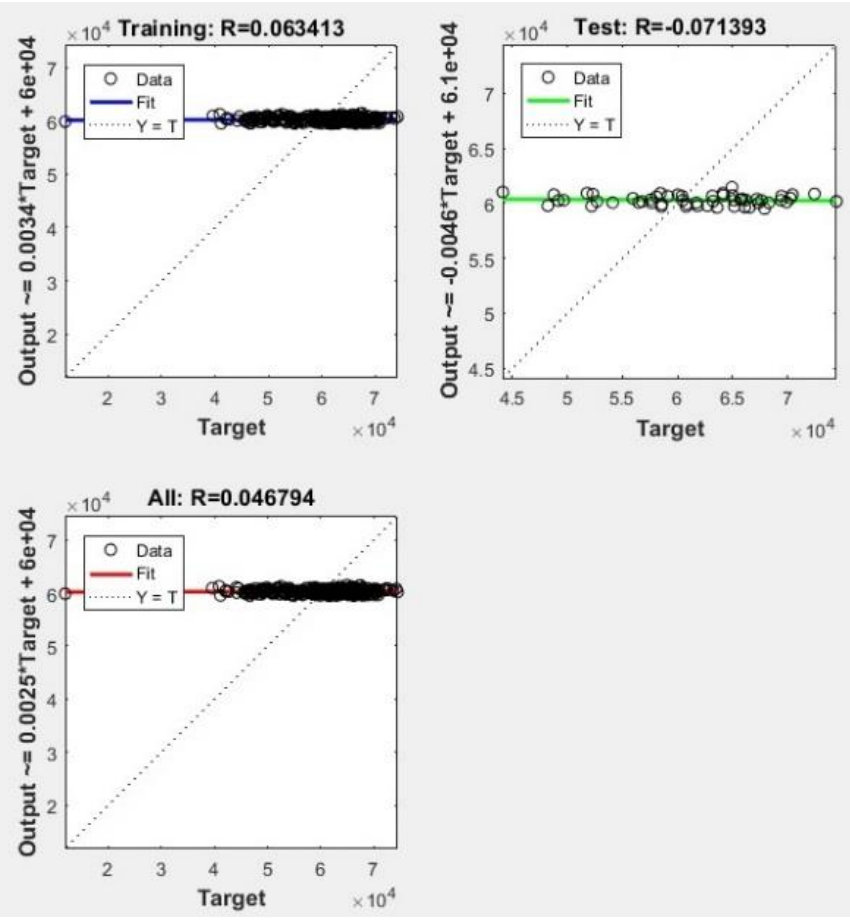

Fig. 5. Regression of Neural Network created function.

\subsection{Comparison}

Results from generated artificial intelligence methods are clearly showing ANFIS advantage for fitting the measured data [14] [15]. Fig. 6 shows graphical presentation of results from ANFIS, ANN and measured data from AMR/AMM.

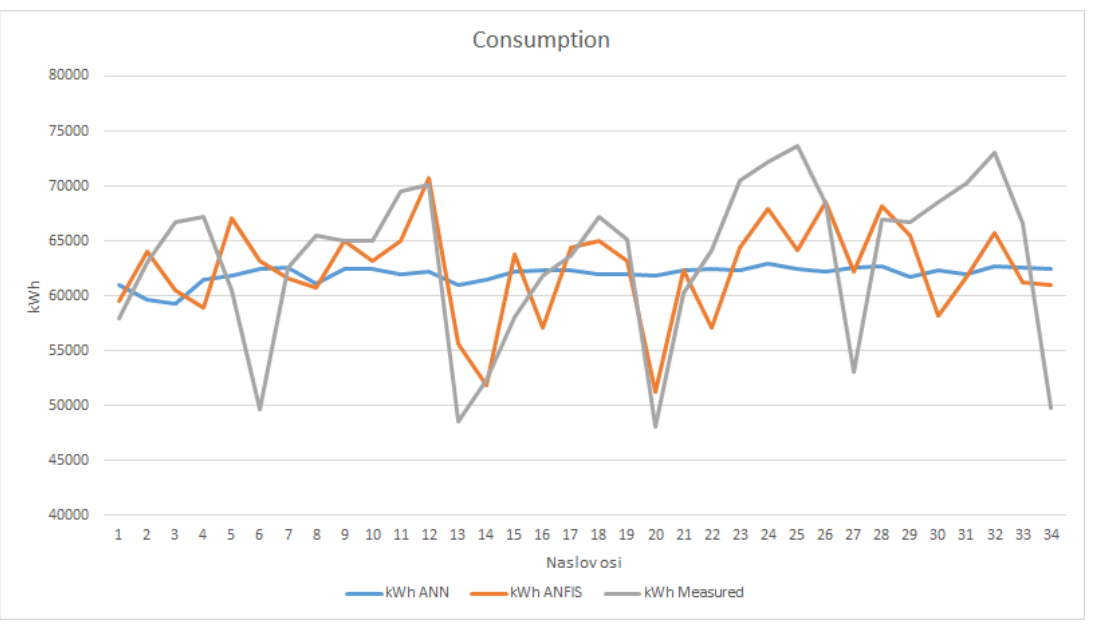

Fig. 6. Compared results from ANFIS and ANN for May 2016, for area Kreševo.

\section{Application structure}

The block scheme presents the structure of the forecasting procedure, as shown in Fig. 7. The diagram shown in Fig. 7 describes the way of process that starts with the web weather service, in this case www.wunderground.com, which gives the needed forecasting maximum, average and minimum temperature for the time period of 1 month in 1 day resolution. By using the developed JAVA application as a standalone runnable .jar, the, data from the web service are transferred to the editable and light textual file with extension .txt. In the Matlab application, the available input data are coming from the generated .txt file and transferred to the online memory of the application. When this is done, the application feedback is the "DONE!!!" note appearing in the application window. The next step is to use the input data for the forecasting consumption concern with the electric energy area, in this case the town of Kreševo, located in Bosnia and Hercegovina. This specific step is done by applying the artificial intelligence method, in this case ANFIS. If successfully done, the user receives the feedback message "DONE!!!". The generated data are stored in output textual file with extension .txt. The entire application is developed using Matlab 64 bit edition GUIDE tool and JAVA code in .jar file developed by using the Eclipse IDE. 


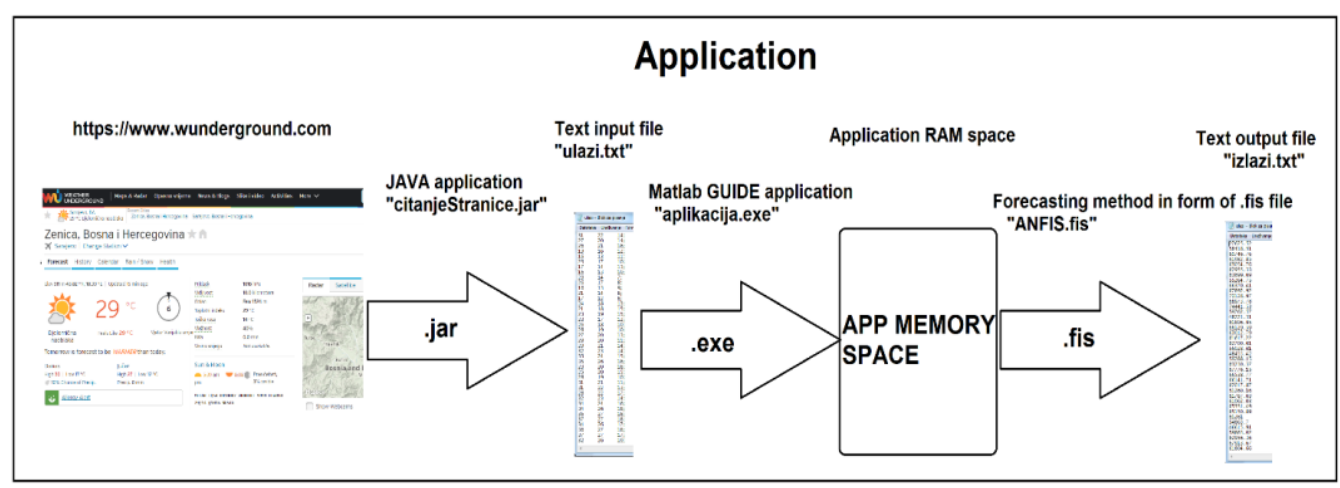

Fig. 7. Block diagram of application for forecasting electric energy consumption.

The purpose of the developed application was to make the method for forecasting the energy consumption mobile and agile, as much as possible, by using the known tools and data sources. It must be noted that today, the availability of the weather forecasting data is huge and the provided data are available and accurate, without the need to further filter or add information. Today the web services, like the one used in this paper, have the high variety of frames to present such data. Those frames are simple to be extracted in form, like textual file shown in this case. By taking into consideration the multiplatform ability of the developed JAVA application module and the strength of the operations available through JAVA, it was a natural choice to develop small application to segment and prepare weather data for Matlab application to integrate with. The only requirement of the application is to be run on the 64 bit OS Fig. 8 shows the developed application outputs by running it from beginning to the end of the entire process, as described before in a few lines.

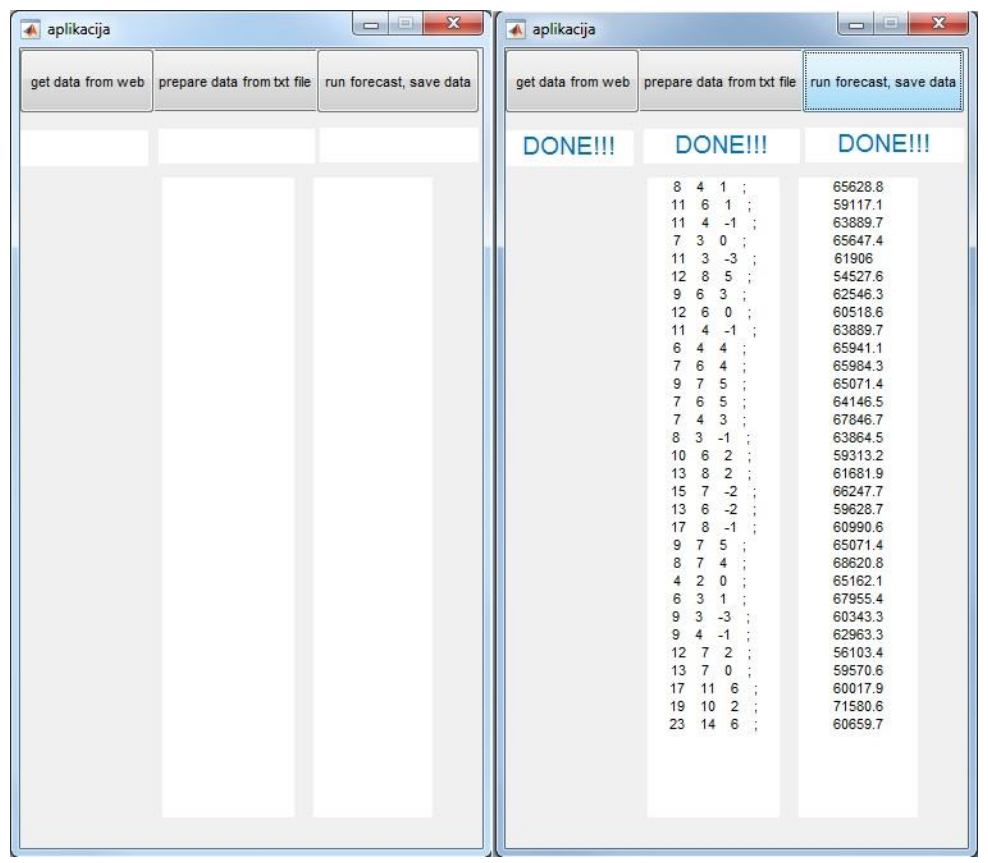

Fig. 8. Left - application at start, right - application at finished run.

\section{Conclusion}

Many times engineers in they work are presented with problem of predicting network consumption which is one of main characteristics of power grid management. Fast results are needed for engineers to work with deadlines [8]. The target of this research was the real life sample, and it is suitable for its ease of measurements, one place to gather all data from field to check error of research. The mobility of the JAVA application, the availability of the weather data and the expertise of the Artificial Intelligence methods are representing the main elements motivating the research described in this paper to give appropriate solution to be accessible, quick and accurate final product. The developed approach was done applying the above-mentioned tools and methods, which are enabled to work with all types of JAVA data, from integer and char to strings and matrix, data from web services, and with most of the AI methods. The data used in this research paper are available for everyone to use, and presented methods are found fast to be implemented with such data. The developed ANFIS algorithm that consist of ANN and with Fuzzy logic, integrated with the MatLab GUIDE application, and customized with the input information in JAVA. This compiled application solution is changeable to any new example from real life and can be extended with any number of input data. The described approach can be applied 
to the distribution network of any voltage level with restriction to the single weather zone. The ANFIS method results show very good accuracy compared to the measured data, thus making the developed mobile application an excellent tool for the fast distribution analysis. Further research plan is to extend the set of knowledge base of ANFIS, and enrich the JAVA application to be customized for the final user, with multiple years of weather reports available to be chosen. The next step of this research will make available to the user different weather areas, with more distribution networks, and more weather history data. All that will be incorporating the present features in a single application, which is the goal for future research.

\section{Acknowledgments}

Creating this paper would be impossible without help of Electric energy Distribution company EP HZ-HB d.d. Mostar, Bosnia and Herzegovina, lending data from their Automatic Meter Reading (AMR) system for purpose of this research.

\section{References}

[1] Abdoulaye Camara; Wang Feixing; Liu Xiuqin (2016). Energy Consumption Forecasting Using Seasonal ARIMA with Artificial Neural Networks Models. International Journal of Business and Management, April 2016, DOI: 10.5539/ijbm.v11n5p231

[2] Nicolás Marrugo; Jorge Ospina; Darío Amaya (2016). Estimated Energy Consumption for a Building Based on Weather and Time Conditions Using Neural Networks. March 2016, DOI: 10.14257/ijca.2016.9.3.02

[3] Qi Zeng; Ning Zhang; Yi Wang; Yuxiao Liu; Chongqing Kang; Zhijian Zeng; Wei Yang; Min Luo (2016). An Optimum Regression Approach for Analyzing Weather Influence on the Energy Consumption. 2016 International Conference on Probabilistic Methods Applied to Power Systems (2016 PMAPS), At Beijing, China, October 2016

[4] Kristopher T. Williams; Juan Gomez (2016). Predicting future monthly residential energy consumption using building characteristics and climate data: A statistical learning approach, Energy and Buildings, June 2016, DOI: 10.1016/j.enbuild.2016.06.076

[5] Deyin Zhao; Ming Zhong; Xu Zhang; Xing Su (2016). Energy consumption predicting model of VRV (Variable refrigerant volume) system in office buildings based on data mining, Energy 102:660-668, May 2016, DOI: 10.1016/j.energy.2016.02.134

[6] Mohammad Ahsan Habib; Md. Hasanuzzaman; M. Hosenuzzaman; Asif Salman; Md Riyad Mehadi (2016). Energy consumption, energy saving and emission reduction of a garment industrial building in Bangladesh, Energy 112:91100, October 2016, DOI: 10.1016/j.energy.2016.06.062

[7] Sasan Barak; S. Saeedeh Sadegh (2016). Forecasting energy consumption using ensemble ARIMA-ANFIS hybrid algorithm. International Journal of Electrical Power \& Energy Systems 82:92-104, November 2016, DOI: 10.1016/j.ijepes.2016.03.012

[8] H.H. Goh; Q.S. Chua; S.W. Lee; B.C. Kok; K.C. Goh; K.T.K. Teo (2015). Evaluation for Voltage Stability Indices in Power System Using Artificial Neural Network, Procedia Engineering Volume 118, September 2015, Pages 11271136, doi:10.1016/j.proeng.2015.08.454

[9] Aleksandr Fedorov; Sergey Zobnin;Viacheslav Shkodyrev; Egor Goloschchapov;Vyacheslav Potekhin (2015). Fuzzy Decision Support In Distributed V2v Systems. Proceedings of the 26th DAAAM International Symposium, pp.0097-0103, B. Katalinic (Ed.), Published by DAAAM International, ISBN 978-3-902734-07-5, ISSN 1726-9679, Vienna, Austria, DOI:10.2507/26th.daaam.proceedings.014

[10] Nyrkov, Anatoliy; Chernyi, Sergei; Zhilenkov, Anton, Sokolov, Sergey (2016). The use of Fuzzy Neural Structures to Increase the Reliability of Drilling Platforms, Proceedings of the 26th DAAAM International Symposium, pp.0672-0677, B. Katalinic (Ed.), Published by DAAAM International, ISBN 978-3-902734-07-5, ISSN 1726-9679, Vienna, Austria,DOI: 10.2507/26th.daaam.proceedings.091

[11] David Walker; Enrico Creaco; Lydia Vamvakeridou-Lyroudia; Raziyeh Farmani; Zoran Kapelan; Dragan Savić (2015). Forecasting Domestic Water Consumption from Smart Meter Readings Using Statistical Methods and Artificial Neural Networks. Procedia Engineering Volume 119, September 2015, Pages 1419-1428, doi:10.1016/j.proeng.2015.08.1002

[12] Xiaoqing Wei; Nianping Li; Wenjie Zhang (2015). Statistical Analyses of Energy Consumption Data in Urban Office Buildings of Changsha, China. Procedia Engineering, Volume 121, October 2015, Pages 1158-1163, doi:10.1016/j.proeng.2015.09.125

[13] Chirag Deb; Lee Siew Eang; Junjing Yang; Mattheos Santamouris (2015). Forecasting Energy Consumption of Institutional Buildings in Singapore. Procedia Engineering Volume 121, October 2015, Pages 1734-1740, doi:10.1016/j.proeng.2015.09.144

[14] Hyojoo Son; Changwan Kim (2015). Forecasting Short-term Electricity Demand in Residential Sector Based on Support Vector Regression and Fuzzy-rough Feature Selection with Particle Swarm Optimization. Procedia Engineering, Volume 118, September 2015, Pages 1162-1168, doi:10.1016/j.proeng.2015.08.459

[15] Fahimifard S.M; Homayounifar M; Mahmood Sabouhi; Moghaddamnia A.R (2009). Comparison of ANFIS, ANN, GARCH and ARIMA Techniques to Exchange Rate Forecasting. Journal of Applied Sciences 9(20), December 2009, DOI: 10.3923/jas.2009.3641.3651 